\title{
Fatores Dietéticos e Síndrome Metabólica
}

\begin{abstract}
RESUMO
O papel da dieta na síndrome metabólica (SM) tem sido estudado em relação a cada um de seus componentes: obesidade, níveis pressóricos elevados, dislipidemia e alterações no metabolismo da glicose. Entretanto, poucos estudos avaliaram os efeitos da dieta na presença da SM como uma entidade clínica independente. O objetivo deste manuscrito foi revisar a importância dos fatores dietéticos e as recomendações dietoterápicas na SM. Estudos recentes demonstraram que o consumo de grãos integrais foi associado negativamente com SM. Já alimentos com alto índice glicêmico foram associados positivamente com resistência à insulina e prevalência de SM. O seguimento de uma dieta mediterrânea foi capaz de reduzir o número de componentes da SM. Também a adoção da dieta DASH melhorou o perfil de todos os componentes da SM. Recomenda-se, para pacientes com SM, um valor energético total compatível com a obtenção e/ou manutenção do peso corporal desejável. O conteúdo de gordura, em especial o consumo de gordura saturada, e colesterol deve ser reduzido e o consumo de grãos integrais, frutas e vegetais, aumentado. Provavelmente as fibras alimentares têm um papel importante no manejo dietoterápico da SM. Novos estudos que avaliem o papel da dieta na presença e no desenvolvimento da SM são necessários. (Arq Bras Endocrinol Metab 2007;51/9:1425-1433)
\end{abstract}

Descritores: Síndrome metabólica; Dietoterapia; Diabetes melito; Fibras dietéticas

\section{ABSTRACT}

\section{Dietary Factors and Metabolic Syndrome.}

The role of diet in metabolic syndrome (MS) has been studied regarding each one of its components: obesity, high blood pressure, dyslipidemia, and abnormal glucose metabolism. However, few studies evaluated the effects of diet in the presence of MS as a unique independent disease. The aim of this manuscript was to review the role of dietary factors and dietary recommendations for MS. Recently some studies demonstrated that intake of whole-grain foods were negatively associated with MS. Foods with high glycemic index were positively associated with insulin resistance and the prevalence of MS. Following a Mediterranean-style diet caused a reduction in the number of MS components. Also, the adoption of the DASH diet improved the profile of all MS components. A total daily energy intake to obtain and/or to maintain a desirable weight is recommended for patients with MS. The fat content, especially from saturated fat, and cholesterol must be reduced and the intake of whole-grain foods, fruits, and vegetables must be increased. Probably, dietary fibers have an important role in the management of MS. New studies to evaluate the role of diet in the presence and development of MS are needed. (Arq Bras Endocrinol Metab 2007;51/9:1425-1433)

Keywords: Metabolic syndrome; Dietary therapy; Diabetes mellitus; Dietary fibers

\section{revisão}

\author{
Thals SteEMBURgo \\ Valesca Dall'Alba \\ JORGE L. GROSS \\ Mirela J. AzeVEDo
}

Serviço de Endocrinologia do Hospital de Clínicas de Porto Alegre, Universidade Federal do Rio Grande do Sul,

Porto Alegre, RS. 
A SÍNDROME METABÓLICA (SM) representa um conjunto de fatores de riscos de origem metabólica que promovem o desenvolvimento de doenças cardiovasculares e de diabetes melito (DM) tipo 2 (13 ). Independentemente do grupo ou entidade que define a SM, os fatores de risco, ou seja, os componentes adotados para sua definição, são praticamente os mesmos (4-6) (tabela 1). Estão incluídos os seguintes componentes: obesidade (especialmente a obesidade abdominal), níveis pressóricos elevados, distúrbios no metabolismo da glicose e hipertrigliceridemia e/ou baixos níveis de HDL colesterol. De acordo com a Organização Mundial da Saúde, a presença de resistência à insulina é necessária para o diagnóstico de SM, mais a presença de dois ou mais componentes. Já para o National Cholesterol Education Program - Adult Treatment Panel III, o diagnóstico de SM é firmado pela presença de três dentre quaisquer dos cinco componentes adotados. Já a obesidade abdominal, associada à presença de dois ou mais componentes, é obrigatória para firmar o diagnóstico de SM de acordo com o International Diabetes Federation.

Outros fatores que também têm sido relacionados à SM são: diminuição do tamanho das partículas de LDL colesterol (LDL pequeno e denso); elevação nos níveis da apolipoproteína $\mathrm{B}$; alterações no estado pró-trombótico (elevações nas concentrações do fibrinogênio e aumento de inibidor-1 do ativador de plasminogênio) e no estado pró-inflamatório (aumento das citocinas: fator de necrose tumoral $\alpha$, interleucina6 e aumento da proteína C-reativa) e elevação dos níveis de ácido úrico (7).

A prevalência da $\mathrm{SM}$ nos Estados Unidos está estimada em 24\%, sendo que acima dos 60 anos de idade esse valor eleva-se para 43,5\% (8). De uma maneira geral, a prevalência da SM vem aumentando (9) e esses achados estão associados com a epidemia de obesidade e DM $(10,11)$. De fato, a SM está fortemente associada ao DM tipo 2 (2), onde cerca de 86\% dos pacientes são portadores da SM (12). Esses dados foram confirmados em estudo realizado em Porto Alegre, RS, onde a prevalência de SM em pacientes com DM tipo 2 foi de cerca de $90 \%$ (13).

O papel da SM como uma entidade clinicamente independente tem levantado muitas questões e controvérsias (14-16). Entretanto, a importância de identificar e tratar esse conjunto de fatores de riscos cardiovasculares é indiscutível. Entre os componentes da SM, a obesidade abdominal é o fator que melhor prediz o risco cardiovascular e de DM (17). A obesidade é também o principal determinante da associação positiva dos níveis de proteína C-reativa com a SM em pacientes com DM tipo 2 (18).

Tabela 1. Definições da Síndrome Metabólica de acordo com diferentes entidades.

\begin{tabular}{|c|c|c|c|}
\hline & OMS, 1998 (4) & NCEP-ATP III 2001 (5) & IDF, 2006 (6) \\
\hline $\begin{array}{l}\text { Diagnóstico de SM } \\
\text { firmado por }\end{array}$ & $\begin{array}{l}\text { Resistência à insulina e presença } \\
\text { de mais } 2 \text { componentes }\end{array}$ & $\begin{array}{l}\text { Presença de } 3 \text { dos } 5 \text { com- } \\
\text { ponentes }\end{array}$ & $\begin{array}{l}\text { Circunferência abdominal } \\
\text { alterada e presença de mais } \\
2 \text { componentes }\end{array}$ \\
\hline \multicolumn{4}{|c|}{ Componentes } \\
\hline Resistência à insulina & $\begin{array}{l}\text { TDG, GJA, DM tipo } 2 \text { ou sensibili- } \\
\text { dade à insulina diminuída }\end{array}$ & - & - \\
\hline Composição Corporal & $\begin{array}{l}\text { Razão cintura-quadril: } \\
\text { Homens }>0,90 \mathrm{~cm} \\
\text { Mulheres }>0,85 \mathrm{~cm} \\
\text { e/ou IMC }>30 \mathrm{~kg} / \mathrm{m}^{2}\end{array}$ & $\begin{array}{l}\text { Circunferência abdominal: } \\
\text { Homens } \geq 102 \mathrm{~cm} \\
\text { Mulheres } \geq 88 \mathrm{~cm}\end{array}$ & $\begin{array}{l}\text { Circunferência abdominal: } \\
\text { Homens } \geq 94 \mathrm{~cm} \\
\text { Mulheres } \geq 80 \mathrm{~cm}\end{array}$ \\
\hline Lipídeos séricos (mg/dl) & $\begin{array}{l}\text { Triglicerídeos } \geq 150 \\
\text { e/ou Homens HDL }<35 \\
\text { Mulheres } \mathrm{HDL}<39\end{array}$ & $\begin{array}{l}\text { Triglicerídeos } \geq 150 \\
\text { e/ou Homens HDL }<40 \\
\text { Mulheres } \mathrm{HDL}<50\end{array}$ & $\begin{array}{l}\text { Triglicerídeos } \geq 150 \\
\text { e/ou Homens } \mathrm{HDL}<40 \\
\text { Mulheres HDL }<50 \text { ou uso de } \\
\text { hipolipemiantes }\end{array}$ \\
\hline Pressão Arterial (mmHg) & $\geq 140 / 90$ & $\begin{array}{l}\geq 130 / 85 \\
\text { ou uso de anti-hipertensivos }\end{array}$ & $\begin{array}{l}\geq 130 / 85 \\
\text { ou uso de anti-hipertensivos }\end{array}$ \\
\hline Glicose sérica (mg/dl) & TDG, GJA ou DM tipo 2 & > 110 (incluindo DM) & > 100 (incluindo DM) \\
\hline Outros & $\begin{array}{l}\text { Microalbuminúria } \\
\text { Excreção urinária de albumina } \geq \\
20 \text { mg/min }\end{array}$ & - & - \\
\hline
\end{tabular}

OMS: Organização Mundial da Saúde, NCEP-ATP III: National Cholesterol Education Program - Adult Treatment Panel III, IDF: International Diábetes Federation, TDG: Tolerância diminuída à glicose, GJA: Glicemia de jejum alterada, DM: Diabetes Melito 
Embora a patogênese da SM não esteja completamente elucidada, seus diferentes componentes possivelmente estão associados à resistência à insulina. $\mathrm{Na}$ SM ocorre uma provável interação entre fatores genéticos, metabólicos e ambientais, incluindo a dieta (19-21).

O papel dos fatores dietéticos na presença e no desenvolvimento da SM não está estabelecido de forma definitiva. Neste sentido, o objetivo deste estudo foi revisar o papel da dieta como fator de risco para a SM e os aspectos relacionados ao tratamento dietoterápico da SM.

\section{FATORES DIETÉTICOS E COMPONENTES INDIVIDUAIS DA SÍNDROME METABÓLICA}

Reduzir o impacto da SM na população significa, antes de tudo, reduzir a incidência da síndrome, antecipando-se ao seu desenvolvimento com medidas preventivas. Essa estratégia é especialmente importante em indivíduos de alto risco, tais como indivíduos obesos ou portadores de DM tipo 2. Em geral, as medidas preventivas relacionadas à alimentação têm sido avaliadas em relação a cada um dos componentes da SM de forma independente. A seguir, serão descritos os principais estudos que analisaram o papel dos fatores dietéticos sobre os componentes individuais da SM: obesidade, pressão arterial elevada, hipertrigliceridemia e HDL colesterol reduzido e hiperglicemia.

\section{Obesidade e dieta}

A obesidade, particularmente a de distribuição abdominal, associa-se a um maior risco cardiovascular decorrente da maior incidência de DM, hipertensão arterial, dislipidemia e da própria SM (22), além de um maior risco de mortalidade em geral (23).

Atualmente, vários tipos de intervenção dietoterápica fazem parte do tratamento não farmacológico proposto para obesidade. Em pacientes com sobrepeso ou obesidade, dietas hipocalóricas que variam entre $800-1500 \mathrm{kcal} /$ dia reduzem aproximadamente $8 \%$ do peso corporal após um período de seis meses de tratamento dietético (24). Uma recente meta-análise demonstrou que no período de um ano uma dieta com baixo conteúdo de gordura, além de promover melhores efeitos no perfil lipídico, foi mais eficaz na redução de peso do que as dietas com baixo teor de carboidratos (25). As dietas muito restritas em calorias, cerca de $<800 \mathrm{kcal} /$ dia, não são recomendadas para uma perda de peso em longo prazo. A restrição energética adotada implica em inadequações nutricionais (24), além de resultar em menor redução de peso quando comparada à dieta hipocalórica tradicional (25). Como visto, as abordagens dietéticas podem variar na prescrição do valor energético total e dos macronutrientes. Entretanto, a diminuição no consumo energético diário é o determinante mais importante para uma perda efetiva de peso. Deve ser lembrado que a associação da dieta com a atividade física aumenta a perda de peso e a diminuição da gordura abdominal (24).

\section{Pressão arterial e dieta}

Entre os fatores ambientais que estão relacionados aos níveis pressóricos, a dieta parece exercer um importante papel tanto em relação à prevenção quanto ao tratamento da hipertensão arterial sistêmica $(1,27)$. Em pacientes hipertensos, o seguimento de uma dieta saudável baseada nos princípios da dieta DASH (Dietary Approaches to Stop Hypertension), rica em frutas, vegetais, produtos lácteos pobres em gorduras (28) e associada a um consumo restrito de sódio $(<5,8$ $\mathrm{g} \mathrm{NaCl} /$ dia) (29), reduz a pressão arterial. O consumo dos alimentos acima descritos, além da restrição de sal, parece ser particularmente importante na redução da pressão arterial. Em 810 indivíduos pré-hipertensos (pressão arterial sistólica de 120-139 mmHg e/ou diastólica de $80-89 \mathrm{mmHg}$ ) ou hipertensos em estágio l (pressão arterial sistólica 140-159 $\mathrm{mmHg}$ e/ou pressão arterial diastólica $90-99 \mathrm{mmHg}$ ), sem uso de anti-hipertensivos, a dieta DASH, quando comparada com uma dieta apenas com redução de sal, ambas acompanhadas de modificações no estilo de vida, promoveu após 18 meses uma redução adicional significativa da pressão arterial de cerca de $1,0 \mathrm{mmHg}$ na pressão arterial sistólica e de $0,4 \mathrm{mmHg}$ na pressão arterial diastólica (30).

\section{Hipertrigliceridemia, HDL baixo e dieta}

A redução dos níveis dos triglicerídeos pode ser alcançada através da redução do consumo de carboidratos de rápida absorção (31). Também o consumo de ácidos graxos ômega-3 derivados de peixes de água salgada foi capaz de reduzir a hipertrigliceridemia (32). A dieta mediterrânea, caracterizada por ser rica em cereais integrais, frutas, vegetais e com elevada proporção de gordura monoinsaturada em relação às saturadas, melhora o perfil lipídico de pacientes com risco cardiovascular elevado. Nesses indivíduos, o seguimento desse tipo de dieta durante um ano reduziu de forma significativa os níveis dos triglicerídeos e aumentou os de HDL-colesterol (33). Além disto, aliado às intervenções dietoterápicas específicas, o consumo moderado de álcool, assim como o aumento da ativi- 
dade física, deve fazer parte do manejo não farmacológico para a redução dos níveis dos triglicerídeos e aumento do HDL-colesterol (27).

\section{Hiperglicemia e dieta}

A hiperglicemia é definida como glicose de jejum alterada, tolerância à glicose diminuída ou DM tipo 2 (6). A importância dos valores de glicose sérica como fator de risco cardiovascular independente é evidenciada já em indivíduos sem DM. Em um estudo populacional com 10.232 indivíduos acompanhados por três anos, foi demonstrado que as concentrações de hemoglobina AlC associaram-se positivamente com a mortalidade por doença cardiovascular e mortalidade total (34).

Dietas que levem em consideração o índice glicêmico (IG) dos alimentos estão entre as propostas atuais para manejo da hiperglicemia, em especial para pacientes com DM. O IG quantifica a resposta glicêmica após ingestão de um alimento comparativamente a um alimento padrão (pão branco ou glicose), cujo IG é considerado como igual a 100. O valor do IG é influenciado pela quantidade e a qualidade do carboidrato do alimento que está sendo avaliado (35). O consumo de alimentos com elevado IG poderia levar a uma exaustão progressiva das células $\beta$ pancreáticas, levando à hiperglicemia (31). Uma dieta com alimentos com baixa carga glicêmica (produto do IG de um alimento e o total de carboidratos deste alimento), associada ao elevado consumo de fibras, particularmente dos cereais integrais, é capaz de reduzir o risco de desenvolvimento de DM (36,37). A importância das fibras sobre o metabolismo da glicose também foi demonstrada em um ensaio clínico controlado realizado em pacientes com DM tipo 2. O elevado consumo de fibras, particularmente as fibras solúveis, teve efeitos benéficos no metabolismo da glicose e dos lipídios (38).

\section{Síndrome metabólica e dieta}

A intervenção dietoterápica faz parte da terapia inicial para o manejo da SM como integrante das alterações de estilo de vida (39). Alguns estudos têm analisado o papel de fatores dietéticos relacionados à SM con-

Tabela 2. Sumário dos principais estudos que avaliaram Dieta e Síndrome Metabólica.

\begin{tabular}{|c|c|c|c|c|c|c|}
\hline Estudo & Delineamento & Grupo em estudo & Definição & Intervenção & Duração & Resultados \\
\hline Freire e cols. (40) & Transversal & $\begin{array}{l}877 \text { indivíduos nipo-brasileiros } \\
\text { (412 homens; } 465 \text { mulheres) }\end{array}$ & NCEP & - & - & $\begin{array}{l}\text { O consumo de gordura total aumentou } \\
\text { enquanto o consumo de ácido graxo poliin- } \\
\text { saturado linolêico reduziu a chance para a } \\
\text { presença de SM }\end{array}$ \\
\hline $\begin{array}{l}\text { Esmaillzadeh e } \\
\text { cols. (41) }\end{array}$ & Transversal & $\begin{array}{l}827 \text { individuos iranianos } \\
\text { (357 homens; } 470 \text { mulheres) }\end{array}$ & NCEP & - & - & $\begin{array}{l}\text { Consumo de grãos integrais foi associado } \\
\text { negativamente com hipertensão arterial, } \\
\text { hipertrigliceridemia e presença da SM }\end{array}$ \\
\hline $\begin{array}{l}\text { Steemburgo e } \\
\text { cols. (42) }\end{array}$ & Transversal & $\begin{array}{l}214 \text { pacientes com DM tipo } 2 \\
\text { (104 homens; } 110 \text { mulheres) }\end{array}$ & IDF & - & - & $\begin{array}{l}\text { Consumo alimentos ricos em fibras solúveis } \\
\text { (grãos integrais e frutas) foi associado negati- } \\
\text { vamente com a presença da SM }\end{array}$ \\
\hline $\begin{array}{l}\text { Sahyoun e cols. } \\
\text { (43) }\end{array}$ & $\begin{array}{l}\text { Transversal } \\
\text { e Coorte }\end{array}$ & $\begin{array}{l}535 \text { indivíduos idosos } \\
\text { (179 homens; } 356 \text { mulheres) }\end{array}$ & NCEP & - & 3 anos & $\begin{array}{l}\text { O consumo elevado de grãos integrais foi } \\
\text { associado negativamente com prevalência } \\
\text { da SM e com redução de risco de mortali- } \\
\text { dade CV }\end{array}$ \\
\hline $\begin{array}{l}\text { Mckeown e cols. } \\
\text { (44) }\end{array}$ & Coorte & $\begin{array}{l}2834 \text { indivíduos } \\
\text { (1290 homens; } 1544 \text { mulheres) }\end{array}$ & NCEP & - & 4 anos & $\begin{array}{l}\text { O consumo de grãos integrais foi associado } \\
\text { negativamente e o consumo de alimentos } \\
\text { com alto IG associado positivamente com a } \\
\text { resistência à insulina e a prevalência da SM }\end{array}$ \\
\hline $\begin{array}{l}\text { Exposito e cols. } \\
\text { (45) }\end{array}$ & $\begin{array}{l}\text { Ensaio clínico } \\
\text { randomizado }\end{array}$ & $\begin{array}{l}180 \text { pacientes com SM } \\
\text { (99 homens; } 81 \text { mulheres) }\end{array}$ & NCEP & $\begin{array}{l}\text { Dieta mediterrânea vs. } \\
\text { dieta AHA }\end{array}$ & 2 anos & $\begin{array}{l}\text { Dieta mediterrânea reduziu número de com- } \\
\text { ponentes da SM }\end{array}$ \\
\hline $\begin{array}{l}\text { Azadbakht e } \\
\text { cols. (46) }\end{array}$ & $\begin{array}{l}\text { Ensaio clínico } \\
\text { randomizado } \\
\text { controlado }\end{array}$ & $\begin{array}{l}116 \text { pacientes com SM } \\
\text { (34 homens e } 82 \text { mulheres) }\end{array}$ & NCEP & $\begin{array}{l}\text { Dieta DASH vs. dieta } \\
\text { para redução de peso } \\
\text { vs. dieta controle }\end{array}$ & 6 meses & $\begin{array}{l}\text { Dieta DASH melhorou acentuadamente o } \\
\text { perfil de todos os componentes da SM (vs. } \\
\text { dieta para redução de peso) }\end{array}$ \\
\hline $\begin{array}{l}\text { Laaksonen e } \\
\text { cols. (47) }\end{array}$ & $\begin{array}{l}\text { Ensaio clínico } \\
\text { randomizado }\end{array}$ & $\begin{array}{c}72 \text { pacientes com sobrepeso } \\
\text { ou obesos } \\
\text { ( } 36 \text { mulheres; } 36 \text { homens) }\end{array}$ & NCEP & $\begin{array}{l}\text { Dieta com modifi- } \\
\text { cação de car- } \\
\text { boidratos: Pão de cen- } \\
\text { teio e massa vs. Pão de } \\
\text { trigo/aveia e batata }\end{array}$ & 3 meses & $\begin{array}{l}\text { Dieta com pão de centeio e massa aumen- } \\
\text { tou a primeira fase de secreção de insulina }\end{array}$ \\
\hline
\end{tabular}

NCEP: National Cholesterol Education Program, SM: Síndrome Metabólica, IDF: International Diabetes Federation, CV: cardiovascular, AHA: American Heart Association, DASH: Dietary Approaches to Stop Hypertension, DM: Diabetes Melito 
siderando-a como uma entidade clínica independente (quadro 2). Em estudos transversais realizados em grupos selecionados de indivíduos, foi demonstrado que o consumo de diferentes tipos de gordura e de grãos integrais foi associado à SM. No Brasil, um estudo realizado na população nipo-brasileira demonstrou que o consumo de gordura total aumentou, enquanto que o consumo do ácido graxo poliinsaturado linolêico reduziu a chance para a presença da SM (40). Em um outro estudo realizado na população iraniana, o consumo de grãos integrais foi negativamente associado com a presença de hipertensão arterial, hipertrigliceridemia e SM (41). É possível que esta associação de SM com os grãos integrais seja devida à presença de fibras. Um estudo recente realizado em pacientes brasileiros com DM tipo 2 demonstrou que o consumo de alimentos ricos em fibras solúveis, representados pelos grãos integrais e frutas, foi um fator de proteção para a presença da SM (42). A importância da ingestão de grãos integrais como protetores para a SM foi confirmada em alguns estudos de coorte. Em indivíduos idosos, a ingestão diária de mais do que três porções de alimentos ricos em grãos integrais foi associada à menor freqüência da SM e a um menor risco de mortalidade por doença cardiovascular (43). No estudo Framingham Offspring Cohort, o maior consumo de alimentos ricos em fibras (particularmente de grãos integrais de cereais) foi negativamente associado com a resistência à insulina e com a menor prevalência da SM. Uma associação positiva foi também observada em relação ao elevado consumo de carboidratos avaliado através do IG dos alimentos (44). Apenas três ensaios clínicos avaliaram a importância da dieta como fator determinante para a SM. A dieta mediterrânea (rica em grãos integrais, legumes, frutas, vegetais, nozes, azeite de oliva e peixes) foi comparada com a dieta recomendada pela American Heart Association (gordura total $<30 \%$ do valor energético total). Ao final de dois anos, o número de componentes da SM foi menor nos pacientes que seguiram a dieta mediterrânea (45). Já em um ensaio clínico de menor duração, a dieta DASH foi mais efetiva na melhora do perfil de todos os componentes da SM (reduções de cintura abdominal, peso, triglicerídeos e níveis pressóricos e aumento do HDL-colesterol) quando comparada com uma dieta controle e com uma dieta hipocalórica para perda de peso (46). Além disso, a alteração na fonte de carboidratos da dieta, provavelmente independente do conteúdo de fibras, foi capaz de modificar a resposta das células $\beta$ pancreáticas à glicose (47). Uma dieta com pão de centeio e massa como principal fonte de carboidratos, comparada com uma dieta rica em pão de farinha de trigo/aveia e batata, aumentou a secreção de insulina na primeira fase após glicose via oral em pacientes com SM e com sobrepeso ou obesidade. Os autores sugeriam que esse efeito sobre a homeostase da insulina possa reduzir o risco de piora da tolerância à glicose e de desenvolvimento de DM.

\section{DIETOTERAPIA NA PREVENÇÃO E NO TRATAMENTO DA SÍNDROME METABÓLICA: RECOMENDAÇÕES}

O tratamento da SM tem como objetivo melhorar a resistência à ação da insulina. Neste sentido, a perda de peso representa a base para o tratamento, pois promove melhora da sensibilidade à insulina reduzindo os riscos para complicações cardiovasculares (48).

A maioria das sociedades internacionais e nacionais não tem ainda diretrizes específicas para a SM como uma entidade clínica independente, e as recomendações são em geral centradas no manejo dos fatores de risco cardiovasculares. Como exemplo, recente publicação conjunta da American Heart Association (AHA) e da American Diabetes Association (ADA) revisa aspectos de prevenção primária de doenças cardiovasculares em pacientes com DM, onde estão incluídas orientações de dieta (49). As diretrizes dietoterápicas apresentadas não diferem de forma apreciável daquelas já publicadas por essas e outras entidades onde existe referência específica à SM $(27,50$ $52,54)$. A seguir, será resumido o posicionamento em relação à dietoterapia no manejo da SM por diferentes entidades.

Segundo a AHA (27), o enfoque principal para pacientes portadores de SM é o controle dos fatores de risco cardiovascular individuais, que pode ser realizado através de modificações no estilo de vida, incluindo a intervenção dietoterápica. É recomendado um consumo calórico balanceado, que, associado à atividade física, permita atingir e/ou manter o peso ideal. Além disto, evitar uma dieta aterogênica, através da redução da ingestão de gordura saturada $(<7 \%$ do valor energético total), de ácidos graxos do tipo trans $(<1 \%$ do valor energético total) e de colesterol $(<300$ $\mathrm{mg} /$ dia), o que pode contribuir de forma efetiva na redução de risco para doença cardiovascular. O aumento do consumo de frutas, vegetais, grãos integrais e peixe é também recomendado.

O National Cholesterol Education Program Adult Treatment Panel III (NCEP-ATP III) (50) fundamenta suas recomendações dietoterápicas em relação à SM no controle dos níveis de LDL colesterol com o 
objetivo de redução do risco de doença cardiovascular. Em relação ao valor energético total, recomenda consumo de $50-60 \%$ de carboidratos, $15 \%$ de proteínas e $25-35 \%$ de gorduras. A ingestão diária de gordura saturada e de colesterol total deve ser menor do que 7\% e do que $200 \mathrm{mg}$, respectivamente. No NCEP-ATP III, é feita uma recomendação específica para o consumo de fibras do tipo solúvel: $10-25 \mathrm{~g} /$ dia.

A ADA (51) ainda não apresenta diretriz nutricional específica para SM. No entanto, uma vez que gorduras saturadas elevam os níveis de colesterol e potencializam a hipercolesterolemia, é recomendado reduzir seu conteúdo dietético a menos do que $7 \%$ do valor energético total e o consumo de colesterol a menos do que $200 \mathrm{mg} /$ dia. A ingestão de gorduras totais não deve exceder 30\% valor energético total. Em relação aos carboidratos, a ADA recomenda diferenciálos quanto à qualidade, isto é, quanto ao índice glicêmico, e não apenas à quantidade total ingerida. Porém, enfatiza que são necessários mais estudos antes de adotar o critério de índice glicêmico como uma diretriz. A ADA também recomenda o consumo de alimentos ricos em fibras alimentares e com baixo IG (14 $\mathrm{g}$ de fibras totais $/ 1000 \mathrm{kcal} /$ dia $)$.

A Sociedade Brasileira de Diabetes (SBD) (52), em recente consenso, considera as modificações no estilo de vida como redução de peso, dieta saudável, exercício regular e cessação do fumo como sendo as principais orientações terapêuticas no tratamento da SM. Não existem recomendações dietoterápicas específicas, porém a dieta mediterrânea (53) é citada como possivelmente associada a uma maior perda de peso.

Tabela 3. Recomendações alimentares para pacientes com Síndrome Metabólica.

\begin{tabular}{|c|c|c|c|}
\hline & Valor diário & Alimentos recomendados & Orientação prática \\
\hline \multicolumn{4}{|c|}{ Valor energético total (VET) } \\
\hline $\mathrm{IMC} \geq 25 \mathrm{~kg} / \mathrm{m}^{2}$ & $800-1500$ kcal & $\begin{array}{l}\text { Dieta balanceada com ênfase no consumo } \\
\text { de alimentos pobres em gorduras; vegetais } \\
\text { do grupo A liberados; preferência por alimen- } \\
\text { tos com baixo índice glicêmico e por carnes } \\
\text { magras }\end{array}$ & $\begin{array}{l}\text { Perder peso é a prioridade; } 3 \text { a } 4 \text { refeições } \\
\text { diárias; alimentos preparados no vapor, forno } \\
\text { ou grelha sem gordura adicional; consumir } \\
\text { alimentos integrais e ricos em fibras e eliminar } \\
\text { álcool }\end{array}$ \\
\hline $\mathrm{IMC}<25 \mathrm{~kg} / \mathrm{m}^{2}$ & $\begin{array}{l}\text { kcal adequadas à atividade } \\
\text { física }\end{array}$ & Ver abaixo & Ver abaixo \\
\hline \multicolumn{4}{|c|}{ Nutrientes } \\
\hline Carboidratos & $50-60 \%$ do VET & $\begin{array}{l}\text { Preferência por cereais integrais ricos em } \\
\text { fibras e alimentos com baixo índice glicêmico }\end{array}$ & $\begin{array}{l}\text { Ingerir diariamente: pão integral ou centeio, } \\
\text { arroz e/ou massa integral; leguminosas e fru- } \\
\text { tas de baixo índice glicêmico (goiaba, maçã } \\
\text { e laranja) e reduzir sucos e açúcar }\end{array}$ \\
\hline $\begin{array}{l}\text { Gorduras } \\
\text { Saturadas } \\
\text { Ácidos graxos trans }\end{array}$ & $\begin{array}{l}<30 \% \text { VET } \\
<7 \% \text { do VET } \\
<1 \% \text { do VET }\end{array}$ & $\begin{array}{l}\text { A maior parte da gordura da dieta deve ser } \\
\text { insaturada; Preferência por carnes de peixes } \\
\text { e aves; lácteos e derivados pobres em gor- } \\
\text { dura; óleos ricos em ácidos graxos poliinsatu- } \\
\text { rados e monosaturados; nozes; amêndoas, } \\
\text { castanhas }\end{array}$ & $\begin{array}{l}\text { Consumir leite e queijos magros, carne de } \\
\text { gado magra; dar preferência a peixe e fran- } \\
\text { go; azeite de canola, girassol ou milho para } \\
\text { cocção e de oliva para temperar; margari- } \\
\text { nas "macias"; evitar biscoitos e bolos industri- } \\
\text { alizados e embutidos }\end{array}$ \\
\hline Colesterol total & $<200 \mathrm{mg}$ & $\begin{array}{l}\text { Carnes de peixes e aves pobres em coles- } \\
\text { terol }\end{array}$ & $\begin{array}{l}\text { Consumir carne de peixe e frango no mínimo } \\
3 \text { vezes por semana; limitar ingestão de } \\
\text { carne de gado até } 2 \text { vezes por semana }\end{array}$ \\
\hline Proteínas & $15 \%$ do VET & $\begin{array}{l}\text { Consumir carnes magras que podem ser } \\
\text { substituídas pelas leguminosas (feijão, lentilha, } \\
\text { grão de bico); o consumo de peixes deve ser } \\
\text { incentivado; leite desnatado e queijo magro }\end{array}$ & $\begin{array}{l}\text { Ingerir leite ou derivados } 2 \text { a } 3 \text { vezes ao dia; } \\
\text { carne magra } 1 \text { vez ao dia }\end{array}$ \\
\hline Sal $(\mathrm{NaCl})$ & 3 a $6 \mathrm{~g}$ & - & $\begin{array}{l}\text { Usar o mínimo de sal no preparo de alimen- } \\
\text { tos; não usar sal na mesa; evitar enlatados e } \\
\text { embutidos. }\end{array}$ \\
\hline $\begin{array}{l}\text { Fibras } \\
\text { Fibras totais } \\
\text { Fibras solúveis }\end{array}$ & $\begin{array}{l}20 \text { a } 30 \mathrm{~g} \\
>10 \mathrm{~g}\end{array}$ & $\begin{array}{l}\text { Alimentos integrais (pão centeio ou integral e } \\
\text { aveia), frutas, vegetais do grupo B e legumi- } \\
\text { nosas alimentos ricos em fibras solúveis }\end{array}$ & $\begin{array}{l}\text { Ingerir diariamente: alimentos integrais, feijão, } \\
\text { frutas ricas em fibras solúveis (laranja, ber- } \\
\text { gamota, mamão papaia, manga), vegetais } \\
\text { do grupo B ricos em fibras solúveis (brócolis, } \\
\text { cenoura, couve-flor) }\end{array}$ \\
\hline Álcool & $\begin{array}{l}1 \text { drinque: mulheres } \\
2 \text { drinques: homens }\end{array}$ & $\begin{array}{l}\text { Não existe recomendação do uso de álcool, } \\
\text { mas na presença de peso adequado, ausên- } \\
\text { cia de hipertrigliceridemia ou outra contra } \\
\text { indicação médica, considerar opção do } \\
\text { paciente }\end{array}$ & $\begin{array}{l}\text { Dar preferência a bebidas não destiladas: } \\
1 \text { drinque }=355 \mathrm{ml} \text { de cerveja, } 120 \mathrm{ml} \text { de } \\
\text { vinho }\end{array}$ \\
\hline
\end{tabular}


A Sociedade Brasileira de Cardiologia (SBC) (54) já apresenta uma diretriz definida para o tratamento não-medicamentoso da SM. O plano alimentar recomendado aos pacientes portadores da SM consiste em fornecer um valor energético total compatível com a obtenção e/ou manutenção do peso corporal desejável. Além disso, o consumo diário de carboidratos, proteínas e gordura total deve ser de $50-60 \%, 15 \%$ e $25-35 \%$ do valor energético total, respectivamente. A ingestão de gordura saturada deve ser limitada a menos do que $10 \%$, a de gordura monoinsaturada, até $20 \%$ e de gordura poliinsaturada, até $10 \%$. O consumo de colesterol deve ser menor do que $300 \mathrm{mg} /$ dia, e para pacientes com valores de LDL-colesterol acima de $100 \mathrm{mg} / \mathrm{dl}$, é sugerido um consumo menor do que $200 \mathrm{mg} /$ dia. É recomendado o consumo de 20-30 $\mathrm{g} /$ dia de fibras totais sob a forma de grãos integrais, frutas, vegetais e leguminosas.

\section{CONSIDERAÇÕES FINAIS}

Os fatores dietéticos podem exercer um papel fundamental tanto nos componentes individuais como na prevenção e controle da SM. Dados recentes associam a presença da SM ao menor consumo de grãos integrais, frutas e vegetais. Existe uma estreita relação entre esses alimentos e as fibras alimentares, e é provável que as fibras do tipo solúvel estejam mais diretamente relacionadas a esses efeitos. A importância das fibras é reforçada pela observação de que o consumo de alimentos ricos em fibras está presente em dietas associadas a uma redução de risco cardiovascular, como a dieta mediterrânea e a dieta DASH. Os mecanismos relacionados aos efeitos benéficos das fibras sobre os componentes da SM não estão ainda completamente esclarecidos. Os efeitos sobre o metabolismo da glicose podem ser mediados por uma absorção e digestão de carboidratos mais lenta, o que resultaria em uma menor necessidade de secreção de insulina após a alimentação (55). De fato, quando pacientes com DM tipo 2 seguem uma dieta rica em alimentos com elevado conteúdo de fibras solúveis, foi demonstrada uma melhora em índices de controle glicêmicos e lipídico associada à redução de hiperinsulinemia (56). As fibras solúveis podem, também, ter um efeito moderado na redução da pressão arterial, como demonstrado em pacientes sem DM (57).

Diferentes fontes e tipos de carboidratos e sua influência na resistência à insulina podem também representar uma alternativa de manipulação de dieta. Neste sentido, a avaliação do papel do IG e da carga glicêmica dos alimentos no contexto da dieta e não apenas de forma isolada deve ser também considerada. Ainda, a avaliação do papel de diferentes tipos de gordura na alimentação usual deve ser analisada em diferentes populações.

O esclarecimento e confirmação de vários aspectos de dieta relacionados à SM discutidos neste manuscrito indicam a necessidade de novos estudos que avaliem a influência de diferentes fatores dietéticos na presença e no desenvolvimento da SM, levando em consideração o papel preponderante da resistência à insulina associada à obesidade abdominal nestes pacientes.

Com base nas evidências existentes sobre o papel da dieta na SM e nas recomendações das principais entidades revisadas neste manuscrito, os autores sugerem recomendações práticas para o manejo dietoterápico da SM (tabela 3).

\section{REFERÊNCIAS}

1. American Heart Association. Diagnosis and management of the metabolic syndrome. An American Heart Association/ National Heart Lung, and Blood Institute/ Scientific Statement. Circulation 2005; 112:2735-52.

2. Wilson PWF, D'Agostino RB, Parise H, Sullivan L, Meigs JB. Metabolic Syndrome as a precursor of cardiovascular disease and type 2 diabetes mellitus. Circulation 2005;112:3066-72.

3. Lorenzo C, Okoloise M, Williams K, Stern MP, Haffner SM. The metabolic syndrome as predictor of type 2 diabetes: The San Antonio heart Study. Diabetes Care 2003; 26:3153-9.

4. Alberti KG, Zimmet PZ. Definition, diagnosis and classification of diabetes mellitus and its complications. Part 1: diagnosis and classification of diabetes mellitus provisional report of a WHO consultation. Diabet Med 1998;15:53953.

5. Executive Summary of the Third Report of the National Cholesterol Education Program (NCEP) Expert Panel on Detection, Evaluation, and Treatment of High Blood Cholesterol in Adults (Adult Treatment Panel III). JAMA 2001;285:2486-97.

6. Alberti KGMM, Zimmet $P$, Shaw J. Metabolic Syndrome - A new world-wide definition. A consensus statement from the International Diabetes Federation. Diabet Med 2006; 23:469-80.

7. Eckel R, Grundy SM, Zimmet P. The metabolic syndrome. Lancet 2005;365:1415-28.

8. Ford ES, Giles VH, Dietz WH. Prevalence of the metabolic syndrome among US adults-findings from the Third National Health and Nutrition Examination Survey. JAMA 2002; 287:356-9.

9. Ford ES, Giles WH, Mokdad AH. Increasing prevalence of the metabolic syndrome among US adults. Diabetes Care 2004:27:2444-9.

10. Olshansky SJ, Passaro DJ, Hershow RC, Layden J, Carnes BA, Brody J, et al. A potential decline in life expectancy in the United States in the $21^{\text {st }}$ century. N Engl J Med 2005;352:1138-45.

11. Zimmet $P$, Alberti KG, Shaw J. Global and societal implications of the diabetes epidemic. Nature 2001;414:782-7.

12. Alexander CM, Landsman PB, Teusch SM, Haffner SM. NCEPDefined metabolic syndrome, diabetes, and prevalence of coronary heart disease among NHANES III participants aged 50 years and older. Diabetes 2003;52:1210-4. 
13. Picon PX, Zanatta C, Gerchaman F, Zelmanovitz T, Gross JL, Canani LH. Análise dos critérios de definição da síndrome metabólica em pacientes com diabetes melito tipo 2. Arq Bras Endocrinol Metab 2006;50:264-70.

14. Gale EAM. The myth of metabolic syndrome. Diabetologia 2005;48:1679-83.

15. Eckel R, Kahn R, Robertson RM, Rizza RA. Preventing cardiovascular disease and diabetes. Diabetes Care 2006; 29:1697-9.

16. Karn R, Buse J, Ferrannini E, Stern M. The metabolic syndrome: Time for a critical appraisal: Joint statement from the American Diabetes Association and the European Association for the Study of Diabetes. Diabetes Care 2005;28:2289-304.

17. Hiller TA, Rousseau A, Lange C, Lépinay P, Cailleau M, Novak $\mathrm{M}$, et al. Practical way to assess metabolic syndrome using a continuous score obtained from principal components analysis - The DESIR Cohort. Diabetologia 2006;49:1528-35.

18. Kahn SE, Zinman B, Haffner SM, Colleen O'Neill, Kravitz BG, $\mathrm{Yu} D$, et al. Obesity is a major determinant of the association of C-reactive protein levels and the metabolic syndrome in type 2 diabetes. Diabetes 2006;55:2357-64.

19. Gropp L. Genetics of the metabolic syndrome. Br J Nutr 2000;83(suppl.1):S39-S48.

20. Lidfeldt J, Nyberg, Nerbrand PC, Samsioe G, Scherstén B Agardh CD. Socio-demographic and psychological factors are associated with features of the metabolic syndrome: the Women's Health in the Lund Area (WHILA) study. Diabetes Obes Metab 2003;5:106-12

21. The National Heart, Lung, and Blood Institute (NHCBI) Family Heart Study. Familial clustering for features of the metabolic syndrome. Diabetes Care 2006;29:631-6.

22. Everson SA, Goldeberg DE, Helmrich SP, Lakka TA, Lynch JW, Kaplan GA, et al. Weight gain and the risk of developing insulin resistance syndrome. Diabetes Care 1998;21:1637-43.

23. Bigaard J, Tjonneland A, Thomsen BL, Overvad K, Heitmann $\mathrm{BL}$, Sorensen TIA. Wait circumference, BMI, smoking, and mortality in middle-aged men and women. Obes Res 2003; 11:895-903

24. Klein S, Burke LE, Bray GA, Blair S, Allison DB, Pi-Sunyer X, et al. Clinical implications of obesity with specific focus on cardiovascular disease. A Statement for professionals from the American Heart Association council nutrition, physical activity, and metabolism. Circulation 2004;110:2952-67.

25. Nordmann AJ, Nordmann A, Briel M, Keller U, Yancy WS, Brehn BJ. Effects of low-carbohydrate vs. low fat diets on weight loss and cardiovascular risk factors. A meta-analysis of randomized controlled trials. Arch Intern Med 2006;166:285-93.

26. Clinical Guidelines on the identification, evaluation, and the treatment of overweight and obesity adults - The Evidence Report. Obes Res 1998;6:51S-209S.

27. American Heart Association. Diet and lifestyle recommendations Revision 2006. A Scientific Statement from the American Heart Association Nutrition Committee. Circulation 2006:114:1-15.

28. Apple LJ, Moore TJ, Obarzanek E, Vollmer WM, Svetkey LP. A clinical trial of the effects of dietary patterns on blood pressure: DASH Collaborative Research Group. N Engl J Med 1997;336:1117-24.

29. Sacks FM, Volmer WM, Appel LJ, Bray GA, Harsha D, Obarzanek $E$, et al. Effects on blood pressure of reduced dietary sodium and the Dietary Approaches to Stop Hypertension (DASH) diet. N Engl J Med 2001;344:3-10.

30. Elmer PJ, Obarzanek E, Volmer WM, Simons-Morton D, Stevens VJ, Young DR, et al. Effects of comprehensive lifestyle modification on diet, weight, physical fitness, and blood pressure control: 18-month results of a randomized trial. Ann Intern Med 2006;144:485-95.

31. Ludwig DS. The glycemic index: physiological mechanisms relating to obesity, diabetes, and cardiovascular disease. JAMA 2002;287:2414-23.

32. Carmena R, Grundy SM. Management of hypertriglyceridemic patients: dietary management of hypertriglyceridemic. Am J Cardiol 1991;68:35A-7.
33. Estruch R, Martinez-Gonzalez MAM, Corella D, Salas-Salvadó J, Rutz-Gutiérrez VR, Covas MI, et al. Effects of a Mediterranean-style diet on cardiovascular risk factors. A Randomized Trial. Ann Intern Med 2006;145:1-11.

34. Khaw KT, Wareham N, Bingham S, Luben R, Welch, Day N. Association of hemoglobin A1C with cardiovascular disease and mortality in adults: The European Prospective Investigation into Cancer in Norfolk. Ann Intern Med 2004;141:41320.

35. Foster-Powell K, Holt SH. International table of glycemic index and glycemic load values: 2002. Am J Clin Nutr 2002;76(1):5-56.

36. Willett W, Manson J, Liu S. Glycemic index, glycemic load, and risk of type 2 diabetes. Am J Clin Nutr 2002;76(suppl):274S-80.

37. Schulze MB, Liu S, Rimm EB, Manson JE, Willett WC, Hu FB. Glycemic index, glycemic load, and dietary fiber intake and incidence of type 2 diabetes in younger and middle-aged women. Am J Clin Nutr 2004;80:348-56.

38. Chandalia M, Garg A, Lutjohann, Bergmann KV, Grundy SM, Brinkley LJ. Beneficial effects of high dietary fiber intake in patients with type 2 diabetes mellitus. N Engl J Med 2000;342:1392-8.

39. Maki KC. Dietary factors in the prevention of diabetes mellitus and coronary artery disease associated with the metabolic syndrome. Am J Cardiol 2004;93(suppl):12C-7.

40. Freire RD, Cardoso MA, Gimeno SGA, Ferreira SRG. Dietary fat is associated with metabolic syndrome in Japanese Brazilians. Diabetes Care 2005;28:1779-85.

41. Esmaillzadeh A, Mirmiran P, Azizi F. Whole-grain consumption and the metabolic syndrome: a favorable association in Tehranian adults. Eur J Clin Nutr 2005;9:353-62.

42. Steemburgo T, Dall'Alba V, Almeida JC, Silva FM, Zelmanovitz T, Gross JL, et al. Intake of fibers from fruits and whole grains has a protective role for the presence of metabolic syndrome in patients with type 2 diabetes. The Endocrine Society's 88th Annual Meeting (ENDO 2006), June 24-27 in Boston, Massachusetts.

43. Sahyoun NR, Jacques PF, Zhang XL, Juan W, Mckeown MN. Whole-grain intake is inversely associated with the metabolic syndrome and mortality in older adults. Am J Clin Nutr 2006;83:124-31.

44. Mckeown MN, Meigs JB, Liu S, Saltzman E, Wilson PWF, Jacques PF. Carbohydrate nutrition, insulin resistance, and the prevalence of the metabolic syndrome in the Framingham Offspring Cohort. Diabetes Care 2004;27:538-46.

45. Esposito K, Marfella R, Ciotola M, Ciotola M, Di Palo C, Giuliano $F$, et al. Effect of a Mediterranean-style diet on endothelial dysfunction and markers of a vascular inflammation in the metabolic syndrome - A randomized trial. JAMA 2004;292:1440-6.

46. Azadbakht L, Mirmiran P, Esmaillzadeh A, Azizi T, Azizi F. Beneficial effects of a Dietary Approaches to Stop Hypertension eating plan on features of the metabolic syndrome. Diabetes Care 2005;28:2823-31.

47. Laaksonen DE, Toppinen LK, Juntunen KS, Autio K, Liukkonen KH, Poutanen KS, et al. Dietary carbohydrate modification enhances insulin secretion in persons with the metabolic syndrome. Am J Clin Nutr 2005;82:1218-87.

48. Tuomiletho J. Cardiovascular risk: Prevention and treatment of the metabolic syndrome. Diabetes Res Clin Pract 2005;682S:S28-S35

49. Buse JB, Ginsberg HN, Bakris GL, Clark NG, Costa F, Eckel R, et al. Primary prevention of cardiovascular disease in people with diabetes mellitus. Diabetes Care 2007;30:162-72.

50. Executive Summary of the Third Report of the National Cholesterol Education Program (NCEP). Detection. Evaluation. Treatment. Adopting healthful lifestyle habits to lower LDL Cholesterol and reduce CHD Risk. In Adults (Adult Treatment Panel III). 2005. Available at: <http://www.nhlbi.nih.gov/ chd>.

51. American Diabetes Association. Nutrition recommendations and intervention for diabetes 2006. A position statement of the American Diabetes Association. Diabetes Care 2006;29:2140-57. 
52. Sociedade Brasileira de Diabetes. Atualização Brasileira sobre Diabetes 2006. Rio de Janeiro: Diagraphic Editora, 2005.

53. Bielsalski HK. Diabetes preventive components in the Mediterranean diet. Review. Eur J Clin Nutr 2004;43(suppl 1):126-30

54. Sociedade Brasileira de Cardiologia. I Diretriz Brasileira de diagnóstico e tratamento da Síndrome Metabólica. Arq Bras Cardiol 2005;84(supl 1):1-27.

55. Riccardi G, Rivellese AA. Effects of dietary fiber and carbohydrate on glucose and lipoprotein metabolism in diabetic patients. Diabetes Care 1991;14:1115-25.

56. Chandalia M, Garg A, Lutjohann, Bergmann KV, Grundy SM, Brinkley LJ. Beneficial effects of high dietary fiber intake in patients with type 2 diabetes mellitus. N Engl J Med 2000;342:1392-8.
57. He J, Streiffer RH, Muntner P, Krousel-Wood MA, Whleton PK. Effect of dietary fiber intake on blood pressure: a randomized, double-blind, placebo-controlled trial. J Hypertension 2004;22:73-80.

Endereço para correspondência:

Mirela Jobim de Azevedo

Hospital de Clínicas de Porto Alegre

Rua Ramiro Barcelos, 2350, Prédio 12,4\% andar

90035-003 Porto Alegre, RS

Fax: (51) 2101-8127 / 2101-8777

E-mail: mirelaazevedo@terra.com.br 